\title{
Formal Analysis and Verification of Agent-Oriented Supply-Chain Management
}

\author{
Muhammad Zubair Shoukat, Muhammad Atif, \\ Imran Riaz Hasrat \\ Department of Computer Science \\ and Information Technology \\ The University of Lahore \\ Lahore, Pakistan
}

\author{
Nadia Mushtaq \\ Department of Software Engineering \\ The University of Lahore \\ Lahore, Pakistan
}

\author{
Ijaz Ahmed \\ Department of Computer and \\ Information Sciences DWC \\ Higher College of Technology \\ Dubai, United Arabe of Emirates
}

\begin{abstract}
Managing various relationships among the supply chain processes is known as Supply Chain Management (SCM). SCM is the oversight of finance, information and material as they move in the flow from different suppliers to manufacturer, wholesaler, retailer and customers. The main problem with such software architecture is coordination and reliability while performing activities. Moreover, continuously changing market makes this coordination challenging. For example failure of production facilities, irregularities in meeting deadlines, unavailability of workers at required times. However, in the Agent-Oriented Supply-Chain Management described in [Mark S. Fox, Mihai Barbuceanu, and Rune Teigen "Agent-Oriented Supply-Chain Management". The International Journal of Flexible Manufacturing Systems, 12 (2000)] the proposed solution claims a remarkable coordination on the basis of an agentoriented software architecture. In this paper, we formally specify architecture and verify it using model checking. We use UPPAAL to formally specify the agents' behaviour involved in SCM. By model-checking, we prove that the given SCM's architecture partially fulfills its functional requirements.
\end{abstract}

Keywords-Supply chain management; agent-oriented supplychain; model checking; formal specification and verification

\section{INTRODUCTION}

Supply chain is a system of organizing activities, people, resources and information involved during the movement of raw material or finished goods from supplier to customer. Managing various relationships among the supply chain processes is known as supply chain management (SCM). Supply chain management $(\mathrm{SCM})$ is the oversight of finance, information and material as they move in the flow from different suppliers to manufacturer, wholesaler, retailer and customers. Supply chain management (SCM) software architecture maintains coordination among and within companies. The main problem with such software architecture is the coordination and reliability while performing activities but the drastically changing market makes the coordination complex.

Supply Chain is not a chain of businesses rather it is a relationship of multiple businesses [1]. It represents a new way of managing the relationship and associated businesses. So, there is need to build standardized methods to put supply chain management $(\mathrm{SCM})$ in practice.

Nirupam Julka et al. in [2] propose a unified framework for monitoring, modeling and management of supply chain. The proposed framework implements various activities of supply chain like production process, enterprise, business knowledge and data. It presents all the activities as an intelligent and unified function. Various software agents are used to compete activities. This framework helps to evaluate and analyze the different business behaviours according to different circumstances faced in supply chain management.

In [3], certain issues regarding agent-oriented supply chain management are investigated and for those issues respective solutions are presented. It is claimed that the proposed solution can handle the complex tasks and interruptions caused by some unexpected events. Our target in this paper is to study the proposed solution for formal specification and verification Formal verification offers a large potential to provide correctness measuring techniques [4]. We apply model checking as formal analysis by using a tool-set UPPAAL.

During the past few years, many automatic verifications and modeling tools for real-time and hybrid structures [5], [6], [7], [8] and [9] have been developed.

The main contribution of this research is a formally described Agent based supply chain management system given in [3] with a set of formal and informal requirements. We prove that the given construction of agent-oriented architecture doesn't meet certain functional requirements. The results are given in the form of message sequence charts.

Structure of the Paper: In Section II, we describe the behaviour of agents which are participants of the agent-orients supply chain management. The behaviour of these participants is formally specified and explained in Section III. Functional requirements are described in Section IV which are specified as formulas in Section IV-A. Results of model-checking are there in Section V. Section VI provides limitations used to develop formal models and we conclude this paper in Section VII.

\section{Agent-Oriented Supply Chain Management SYSTEM}

In Fig. 1, the basic architecture is shown which tells about the customer conversation with the logistics agent. The process starts when a customer agent place an order, the logistics agent receives the proposal and acknowledges the customer about the received order. Logistics receives the order and 


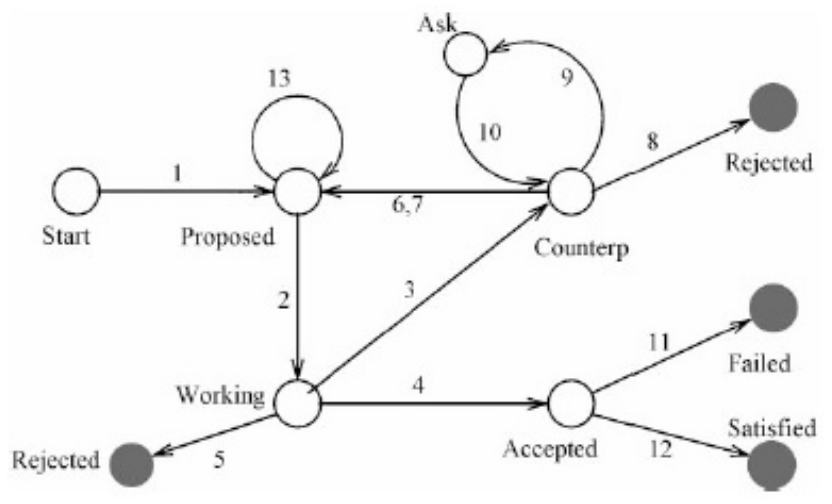

Fig. 1. Customer conversation [3].

tries to decompose the order into different activities if the order decomposed the next process starts if decomposition not possible the process ends. In case of decomposition the next process will be the ranking of contractors on the bases of the activities that are formed after decomposing the order. This process contains two steps: one is formation of large teams and the other is formation of small teams.

In large teams all the contractors that are interested in performing the activities are involved each activity have at least one contractor. After this small teams formed in which one contractor assigned to the one activity. In this stage small team should work cooperatively and inform to the logistics if they have any problem that make it impossible for them to complete their task or activities assigned to them. In case of decomposition is failed, the logistics acknowledges negatively so that the customer may change the proposal. The possible changes can be on the basis of requirements, time or contractors' availability. If logistics again cannot handle a modified proposal then it goes to rejected state.

After the work completion Logistics hand over the work to the customer and customer state is accepted then. If the customer is satisfied then feedback is provided by going on satisfied state. In this way, multiple agents can be considered while placing an order in the agent based supply chain management system.

\section{A. Customer and Logistics Agents}

- Customer Agent: The customer agent sends proposal to the logistics agent and goes to working state. After the processing a proposal the logistic agents acknowledge to the customer agent. The customers may go to a rejected state or can ask for counter proposal if the order cannot be decomposed. If customer satisfied then it goes to satisfied state otherwise on failed state.

- Logistics Agent: The logistics agent receives the proposal and works on it. Logistics agent also informs the customer that working has been initiated on the proposal. Logistics agent decomposes the order or proposal, rank the contractors and also creates the teams that are able to perform the activities according to customer need. Logistics agent also negotiates with customer if there is delays in work or if the decom- position not possible logistics negotiate with different proposal.

\section{Formal SPECIFICATIONS}

Our formal specification in UPPAAL covers the following participants or processes, i.e. the Customer, the Logistics and the Small Team. The main process is the Logistics process. The logistics process receives and sends messages to the other processes to communicate with them. The customer can sends order to any logistics process using handshaking channels. After reception of order the logistics decomposes the order and sends it to the small team. The small team communicates with logistics process and committed to complete that task after checking its schedule. We gives a brief description of the formal specifications model checking of main processes in our explanation of the architecture.

We specify all the concurrent processes of Agent-Oriented Supply-Chain Management. The Customer, Logistics and Small Team are the processes or participants in the given model. These processes of the software architecture are modeled as parallel processes.

\section{A. Channels}

This software architecture uses thirteen channels. To model the functionality of Agent-Oriented Supply-Chain Management the following channels are used for one-to-one communication or for broadcasting:

1) Proposal: This channel is used by a customer to send some proposal to logistic agents.

2) Order: If proposal is accepted then this channel is used place order for selected items.

3) Reject; This channel is used to acknowledges a customer if some order can be processed or not.

4) Success: A completion of order is conveyed through this channel.

5) Failed: A team uses this channel if some order cannot be completed with certain time.

6) Complete: If a task is completed successfully then an acknowledgment is sent by a small team through this channel.

7) NegT1: This channel is used to send task to the small team 1 by the logistics.

8) NegT2: This channel is used to send task to the small team 2 by the logistics.

9) Commited: This channel is used to send acknowledgment to the logistics by the small team if the team is interested and willing to work.

10) Alternative: This channel is used to send acknowledgment to the logistics by the small team if the team has some issues in the proposal and needs alternative which is received from the logistics.

11) NewT1: This channel is used to send task to the small team 1 after the new contractor is assigned to the existing task.

12) NewT2: This channel is used to send task to the small team 2 after the new contractor is assigned to the existing task.

13) Change: This channel is used to send acknowledgment to the small team if the new contractor is not available for the existing task. 


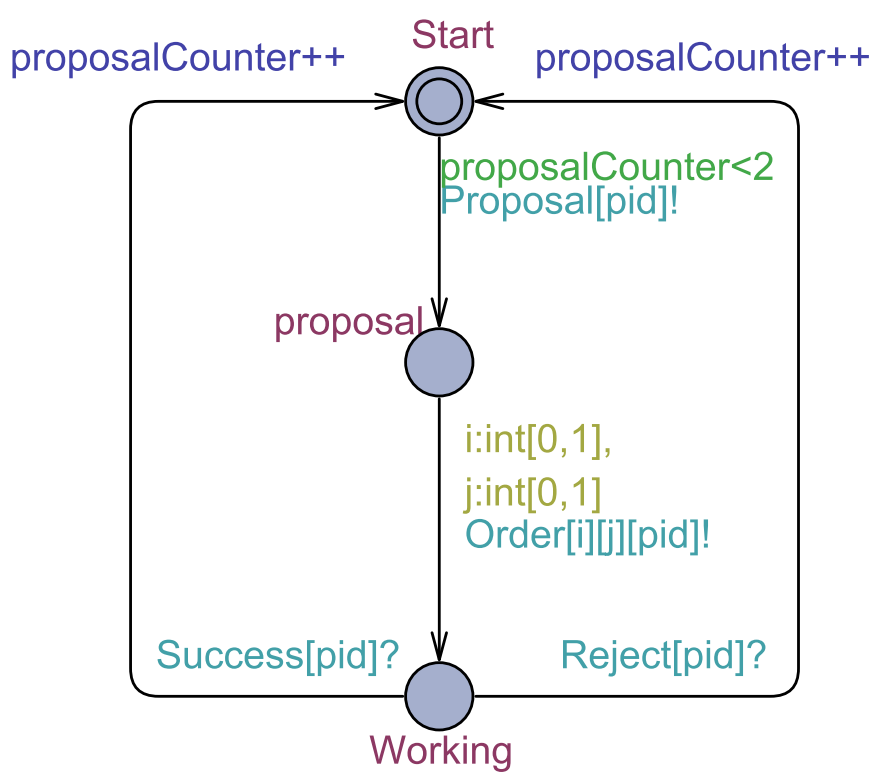

Fig. 2. The customer process.

\section{B. Behaviour of a Customer Process}

In Fig. 2, the automaton for Customer process is illustrated. The initial state is named as Start. The Customer process has three states. The first state is Start state, second is Proposal state and the third state is Working state. There are four major actions in this process described below:

1) Sending proposal to the Logistics.

2) Sending Order to the Logistics and waiting for the response.

3) Going to the start state through rejected path if response is negative from Logistics.

4) Going to the start state through success path if order is successfully completed.

First of all, the channel Proposal transfers a value to some logistic agent which is originated by a customer process. The logistic agents receives these values while synchronization of channel Proposal $[x]$. Here 'x' represents process ID (pid), i.e., the customer ID sending a proposal.

Secondly, after sending proposal the customer sends order using channel Order[2][2] which is received by the logistics at channel Order[i][j][cus_id] and goes to the Working state. There are two values ' $\mathrm{i}$ ' and ' $\mathrm{j}$ ' that are sent by the Customer for the activities that a customer needs. If $i=0$ and $j=1$ the Customer needs activity $j$, if $i=1$ and $j=0$ the customer needs activity $i$ and if both $i$ and $j$ are 1 the customer needs both the activities.

At the end if the customer receives error message from the logistics that the order cannot be processed or teams fail to work then it goes to Start state using Reject channel, if the work is successfully done it goes to Start state using Success channel. On initial state means that it is ready for the next proposal. There is a counter proposalCounter for the proposals sent by a Customer.

\section{Automaton for the Logistics Process}

In Fig. 3 the automaton for Logistics process is illustrated. The initial state is named as Start. There are five major actions in this process described below:

1) Receiving proposal from the Customer and decompose it.

2) Forming small team of contractors that will execute the activities.

3) Providing alternative if small team has issue in the order.

4) Providing alternative contractor if one team needs alternative and other one ready to work.

5) Providing alternative contractor if one team fails to complete its work and other one successfully completed work.

The Fig. 4 shows that the Logistics process receives proposal using synchronization channel Proposal $[x]$. These values are process ID of Customer describes that which customer sends order. After receiving proposal the Logistics receives order from the Customer using channel Order[i][j][cus_id]?. There are two values ' $\mathrm{i}$ ' and ' $\mathrm{j}$ ' that are received by the Logistics are the activities that customer needs. If $i=0$ and $j=1$ the Customer needs activity $j$, if $i=1$ and $j=0$ the Customer needs activity $i$ and if both $i$ and $j$ are 1 the Customer needs both the activities.

After receiving order a Logistic agent tries to rank contractors according to the activities a Customer demands. For example if customer needs A1 activity then contractor that can perform A1 activity is not available then the order is rejected and Logistics goes to Start state, ready to receive new order and acknowledges the Customer. Similarly, if Customer needs A1 and A2 activities contractors for both activities should be available. If contractors successfully ranked Logistics assign activities to contractors and goes to ContractorRanked State.

Fig. 5 shows the next part of the Logistics process. After ranking the contractors Logistics waits for the response from the small team whether or not they will accept the contract. This is done by sending each activity to that small team which is available and willing to do work. For this purpose NegT1[pid][0]! and NegT2[pid][1]! channels are used for SmallTeam(0) and SmallTeam(1), respectively where [pid] is the process id of Logistics sending order and [0] and [1] values describe the pid of SmallTeam to which Logistics are sending order. The response from the SmallTeam can be of three types Logistics receives it on SmallTeam state which is as follows:

1) Both the Small Teams are ready to do work or committed.

2) Small Team 1 needs alternative and Small Team 2 ready to do work.

3) Small Team 1 ready to do work and Small Team 2 needs alternative.

If both the Small Teams are ready to do work. Then the Logistics receive the response using channels Commited[pid][c]? from both teams. Commitcount ++ is used to count the commit response, if the value in Commitcount is 2 it means both teams committed in case of Customer needs one activity the value of Commitcount will be 1. If Small Team 1 needs alternative 


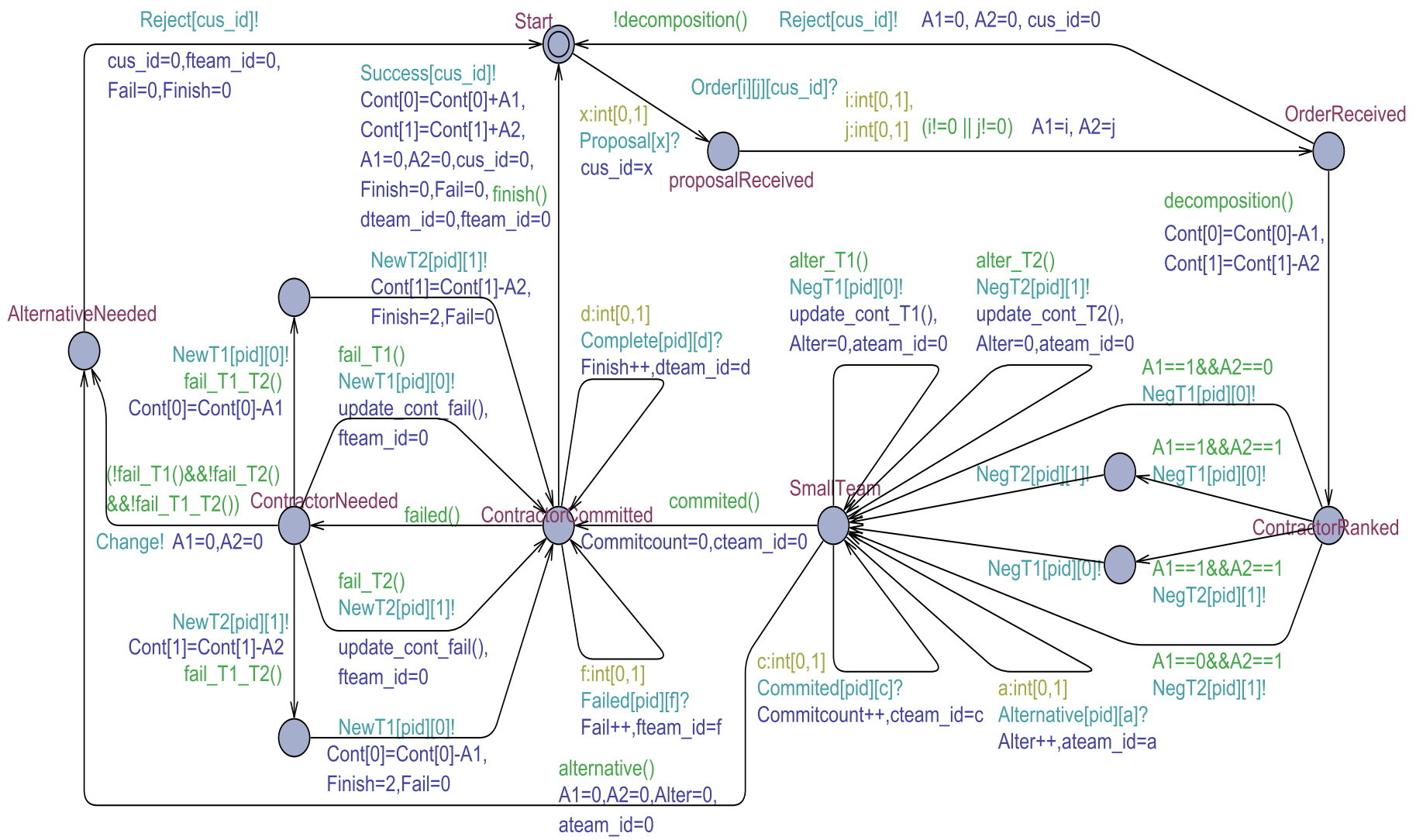

Fig. 3. The logistics process.

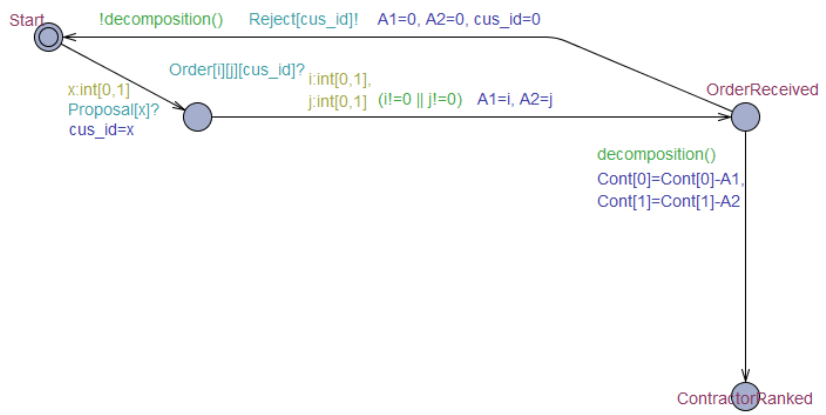

Fig. 4. The logistics process (a).

and Small Team 2 ready to do work or vice versa then Commited[pid][c]? for committed and Alternative[pid][a]? for alternative response is used. Commitcount ++ used to count the commit response and Alter++ used to count the alternative response.

When one team is committed and other needs alternative then Logistics checks for the available contractors willing to work and assign activity for which Small Team needs an alternative. This is done by using NegTl[pid][0]! and NegT2[pid][1]! for Small Team 1 and Small Team 2, respectively.

Fig. 6 shows the next procedure after small team formation. If both the teams need alternative in case Customer needs

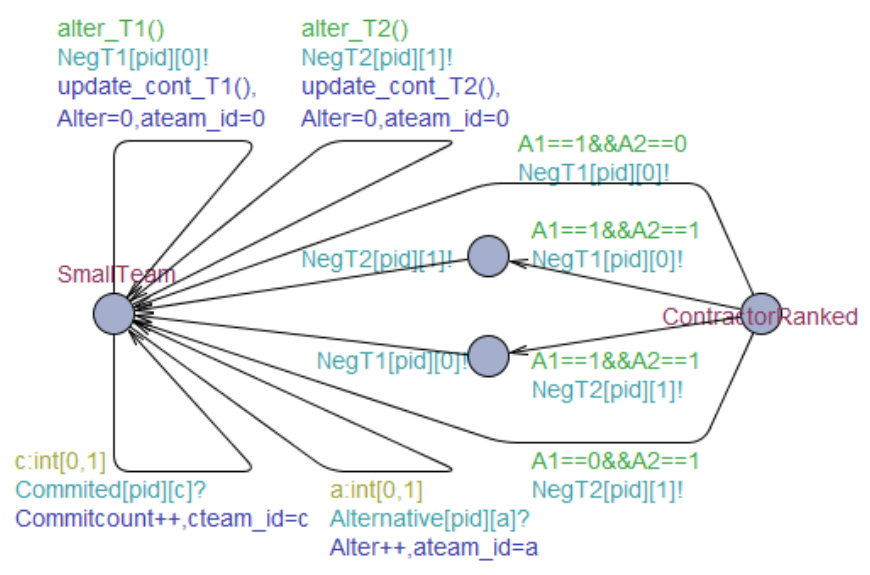

Fig. 5. The logistics process (b).

both activity then process goes to AlternativeNeeded state. Moreover, it goes to Start state for negative acknowledgment to customer using channel Reject[cus_id]! after which it becomes ready for receiving new order. In case of small Teams are committed to work then process goes to Contractorcommitted state. At this state Logistics checks whether small teams have complete their work or not. If the teams complete their work successfully then the respective logistic agent goes to start state using channel Success[cus_id] and acknowledges the Customer accordingly. The number of failed teams are counted 


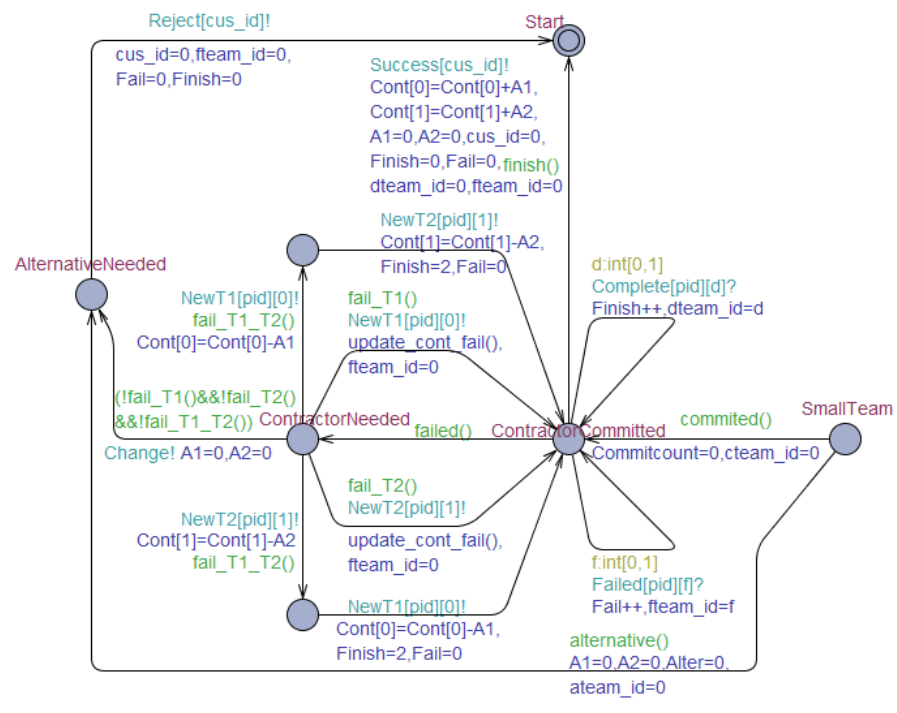

Fig. 6. The logistics process (c).

\section{by Fail.}

When one team is successful and other team fails then Logistics checks for the available contractors that are willing to do work and assign activity for which Small Team fails. This is done by using NewT1[pid][0]! and NewT2[pid][1]! for Small Team 1 and Small Team 2, respectively. If the contractor is available activity assigns to that contractor and after completing work Logistics process goes to ContractorCommitted state and further more at Start state. In case of contractors are not available then process goes to AlternativeNeeded using channel Change! this transition further more goes to start state using channel Rejected[cus_id]!.

When we use UPPAAL system models, functions can be declared within the procedure or process. We can pass parameters in functions and functions can also have return type. The Logistics Process have various functions and are used at different transitions to perform its functionality.

1. Decomposition(): function used as guard and checks the contractor against activities. $A 1$ and $A 2$ are the activities. If customer needs both activities vales of $A l$ and $A 2$ will be 1 , if customer needs one activity then the value of $A 1$ and $A 2$ will be 1 according to the activity that customer needs. This guard prevents to take action if the contractors will not available against the activity which customer needs and take action that goes to start state so logistics can receive new order.

2. Committed(): function is also used as guard and checks the small teams response. If customer needs both the activities then both the teams should be committed to work if not, guard will prevent to goes to ContractorCommitted state. If customer needs one activity then the small team against that activity should be committed. This Function uses an integer variable CommitCount for counting the response form the the teams and compares with number of teams and activities.

3. Alternative(): function is also used to check the small team response. It works same as committed() function but the difference is that in case of both activities, if both the teams need alternative this guard will allow to go to AlternativeNeeded state through alternative transition. And if customer needs one activity then the small team against that activity can ask for alternate. This Function uses an integer variable Alter for counting the response form the the teams and compares with number of teams and activities.

4. Alter_T2(): function is used to check the availability of contractors. If the customer needs both the activities and 1st team committed and 2 nd team needs alternative, then contractor for 2 nd team should be available otherwise this guard will prevents to take further action and wait for the availability. Statement $\operatorname{Cont}[1]_{i} O$ checks the availability. This function uses Commitcount and Alter variables to check which teams needs alternative.

5. Alter_T1(): function is also used to check the availability of contractors. If the customer needs both the activities and 1st team needs alternative and 2 nd team is committed, then contractor for 1st team should be available otherwise this guard will prevent to take further actions and waits for the availability. Statement $\mathrm{Cont}\left[\mathrm{O}_{i} \mathrm{O}\right.$ checks the availability. This function uses Commitcount and Alter variables to check which teams needs alternative.

6. Finish(): function is used when small team complete their work successfully after commitment. In case of customer needs one activity, variable Finish value will be 1 and function allows to finish the work and Logistics goes to Start state to take new order. If customer needs both activities value of Finish will be 2 .

7. Failed(): function is used if the small team fails to complete work after commitment. Both the teams can be failed or may be one team fail and other complete the task then this function will allows to take action and goes to ContractorNeeded state. Fail and Finish variables are used to check which teams are failed or has completed their work. If value of Fail is 2 then both the teams failed, if value of Fail and Finish is 1 then one team has completed and other has finished the work.

8. Fail_T2(): function is used to check the availability of contractors in case of one team finishes its work and other team completes its work successfully. If the customer needs both the activities and 1st team finished work and 2nd team failed, then contractor for 2 nd team should be available for replacement otherwise this guard will prevents and goes to AlternativeNeeded state. In case if a customer needs only 2nd activity and small team fails to complete work then contractor against that activity should be available for replacement. Statement $\operatorname{Cont}[1]_{6} O$ checks the availability. This function uses fteam_id variable to check which teams is failed.

9. Fail_T1(): function works same as fail_T2() difference is that if the customer needs both the activities and 1st team failed and 2nd team finished, then contractor for 1st team should be available for replacement otherwise this guard will prevents and goes to AlternativeNeeded state. And if customer needs only 1st activity and small team fails to complete work then contractor against that activity should be available for replacement. Statement Cont $[0]_{6} O$ checks the availability.

10. Fail_T1_T2(): function is used if customer needs both activities and both are failed to complete their work after 


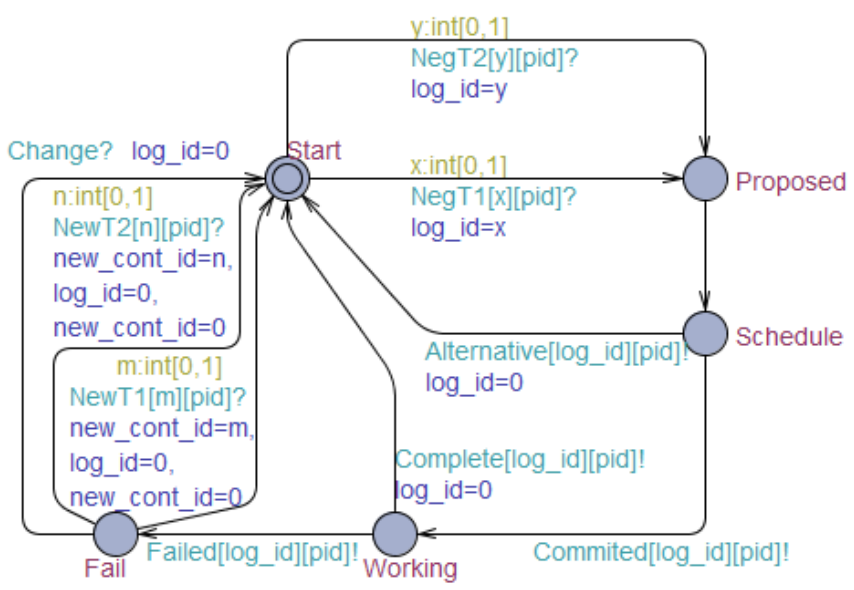

Fig. 7. The small team process.

commitment then both contractors for 1 st team and 2 nd team should be available for replacement otherwise this guard will prevents and goes to AlternativeNeeded state. Statement Cont $[0]_{i} O$ and $\operatorname{Cont}[1]_{i} O$ checks the availability.

11. Update_cont_Tl() and update_cont_T2(): functions are used to update contractors. If customer needs both activities and one team is committed and other needs alternative. The function used cteam_id, ateam_id and Alter variables to check which team is committed and which one needs alternative.

12. Update_cont_fail: function is used to update contractors in case of customer needs both activities and one team has completed its work successfully and other team failed to work. This function uses same variables as previous functions.

\section{The Automaton for the Small Team Process}

Fig. 7 the automaton for small team process is illustrated. The initial state is named as Start. The Logistics process has five states. The first state is Start state, second is Proposed, third is Schedule, fourth is Working and the fifth state is Fail state. There are four major actions in this process described below:

1) Receiving proposal from the logistics.

2) Sending acknowledgment to the logistics if needs some changing or alternative proposal.

3) Sending acknowledgment to the logistics if ready to perform activity and goes to Working state.

4) If fails to complete activity goes to fail state and waiting for new contractor who is willing to complete that activity.

The channel NegT1[x][pid]? and NegT2[y][pid]? receives value from the logistics process, received values are the tasks assigned to the team 1 and team 2 received from channels NegT1[x][pid]? and NegT2[y][pid]?, respectively. The small team checks its schedule if team has no issue and willing to work. Then this small team acknowledges the logistics using Commited[log_id][pid]! channel to go to Working state. If there are issues in the proposal like small team has not enough time or could not perform that activity on time, small team acknowledges the logistics that it needs alternative for that task using Alternative[log_id][pid]! channel and goes to Start state for receiving new or alternative proposal.

After committing small team starts working on the task. If small team fails to complete its task it sends negative acknowledgment to the logistics that it needs new contractor and goes to Fail state waiting for new contractor to be assigned by the logistics and this is done by using Failed[log_id][pid]! channel. If the contractor is available and willing to work small team is assigned that contractor using NewTI[m][pid]? and NewT2[n][pid]? channels for team 1 and team 2, respectively otherwise goes to Start state after receiving response from the logistics using Change? channel.

\section{Functional ReQuiREMENTS}

According to [3], we extract the following functional requirements:

R1: Deadlock freedom. No deadlock when a customer needs to places an order. In other words, deadlock can occur only when there are no more orders.

R2: If customer sends order, logistic agents eventually acknowledge it.

R3: A customer is in working state after paying an order.

R4: If logistics agent is in OrderReceived state if it receives an order.

R5: $\quad$ Every order decomposed by some logistic agent results in formulating a small team.

\section{A. Formal Specification of Requirements}

In this section, we describe formal specification of the requirements. The customer process sends order and then increases its counter, i.e., known as proposalCounter. This increment continues up to two it means the customer can send maximum 2 orders. So, according to the R1 requirement, there is deadlock only when there are no more orders to send by the customers. The formula of $\mathrm{R} 1$ requirement is given below.

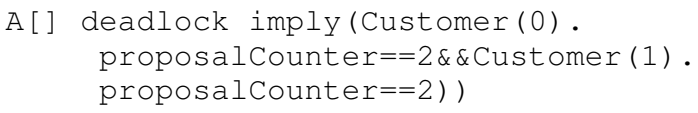

When customer sends order the logistics agent receives and acknowledges it with a message either the given order is workable or not. The formula of to represent this requirement is:

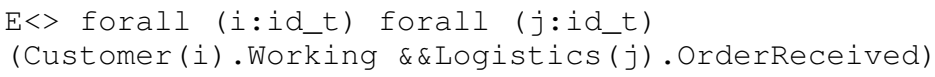

Formula describes that Customer(0) and Customer(1) sends proposal to Logistics(0) and Logistics(1) and vice versa. The logistics acknowledges the customer.

When customer sends order the logistic agents receives and acknowledges the customer at that time customer goes to Working state. For example, when customer(0) sending order definitely goes to working state. The formula of this requirement is.

forall (i:id_t) Customer(i).proposal -->

Customer(i).Working 
According to the $\mathrm{R} 4$ requirement, when a logistics agent receives proposal it goes to OrderReceived state. The formula of the requirement is given below.

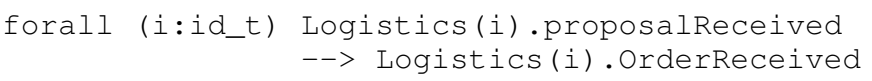

According to the R5 requirement, every order decomposed by some logistics agent formulates a small team. The formula of this requirement is given below.

forall (i:id_t) Logistics(i). ContractorRanked $-\rightarrow$ Logistics(i). SmallTeam

\section{RESUlTS}

To analyse features specified in the above section, we use the verifier, a feature of UPPAAL model checker. Ultimate results are derived in query section of verifier feature and presented in Table I. In query section, the feature is written and its consequences are to be revealed in the status section. The outcomes are in the form of "Satisfied" and "Not Satisfied" of property. We verify our system model for,

Total Number of Processes $=3$

Order Sending Limit $=2$

Activity Demand Limit $=2$

TABLE I. RESULTS

\begin{tabular}{|c|l|c|}
\hline \hline Requirement & Status & Computational Time \\
\hline \hline R1 & Not Satisfied, 131 states & $0.125 \mathrm{sec}$ \\
\hline R2 & Satisfied, 28,180 KB & $0.015 \mathrm{sec}$ \\
\hline R3 & Satisfied, 138 states & $0.539 \mathrm{sec}$ \\
\hline R4 & Satisfied, 1623 states & $0.562 \mathrm{sec}$ \\
\hline R5 & Not Satisfied, 32,204 KB & $0.032 \mathrm{sec}$ \\
\hline
\end{tabular}

$\mathrm{R} 1$ : This requirement is violated and not satisfied. According to the requirement system should be deadlock free or deadlock can occur only when there are no more orders to send. But there is a scenario in which this requirement is not satisfied. When a small team needs alternative there is no more contractor available against that activity at that state deadlock occurs. The counter example for requirement R1 generated by UPPAAL is shown in Fig. 8 .

$\mathrm{R} 5$ requirement is not satisfied and according to this requirement upon decomposing an order by logistic agents, small team is formed. If a customer needs both activities then upon decomposition if one small team needs alternative but there contractors are unavailable pertaining to that activity then small team is not formed, so this requirements is not satisfied. The counter examples for the requirement is shown in Fig. 9.

\section{LimitATIONS AND CHALLENGES}

There are some obstructions for authentication of intended AgentOriented Supply-Chain Management. We restrict the number of orders to two. We also restrict the number of activities to two and the contractors against those activities. A customer can send maximum two orders and demands for maximum two or minimum one activity. These limitations reduce the state space because the model generates a huge state space. The machines are used in our verification have limited resources for memory and speed. These limitations are also used due to limited memory of machine. The machine can crash during execution of query verification phase. We perform some computations on the machine with 4GB RAM, core i3(3rd Gen) Laptop.

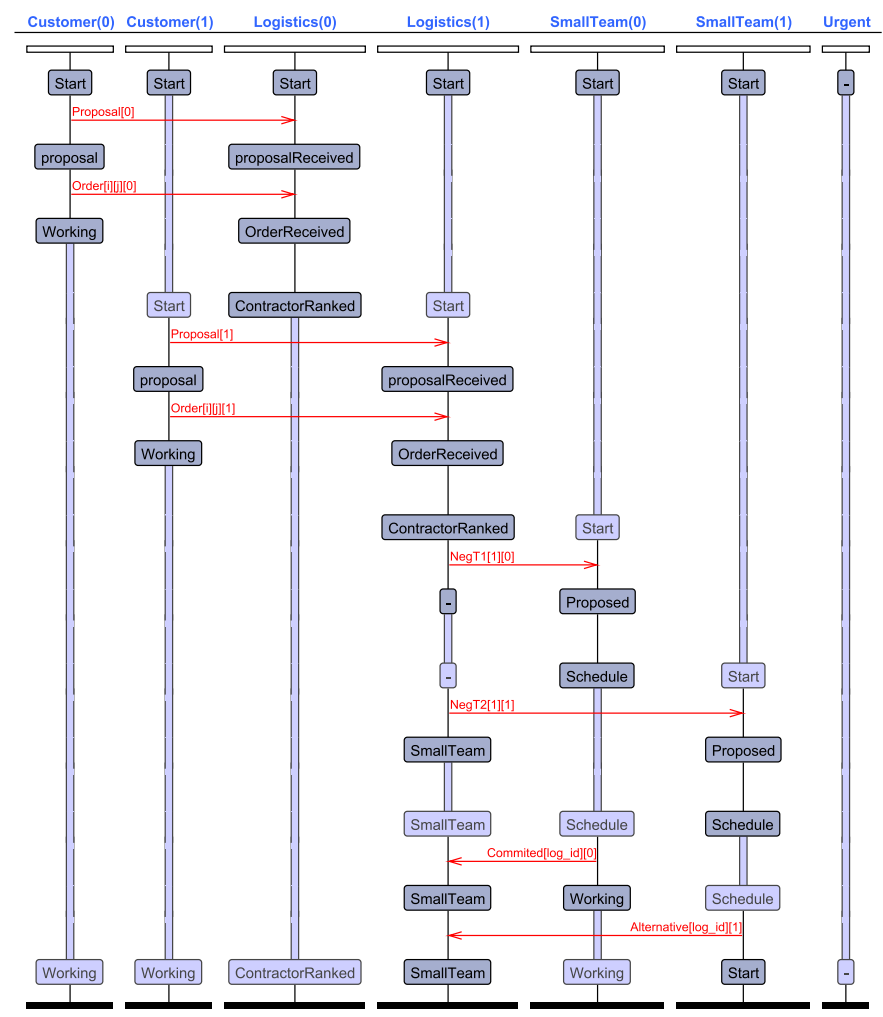

Fig. 8. Trace for requirement R1.

\section{CONCLUSION}

We formalized Agent-Oriented Supply-Chain Management as specified in [3] in UPPAAL model checker. We then formalized functional requirements of the architecture and verified them by model checking. Results show that the given architecture partially fulfills its functional requirements. Proof the results are presents in the form for message sequence charts. The given protocol is verified with limited number of logistic agents, orders and customers.

\section{REFERENCES}

[1] M. C. C. Douglas M. Lambert, "Issues in supply chain management," Industrial Marketing Management, vol. 29, p. 19, 2000.

[2] I. K. Nirupam Julka, Rajagopalan Srinivasan, "Agent-based supply chain management-1: framework," Computers and Chemical Engineering, vol. 26, p. 15, 2002.

[3] R. T. Mark S. Fox, Mihai Barbuceanu, "Agent-oriented supply-chain management," in The International Journal of Flexible Manufacturing Systems, 12. Kluwer Academic Publishers, 2000, pp. 165-188.

[4] C. Baier, J.-P. Katoen et al., "Principles of model checking, vol. 26202649," MIT Press Cambridge, vol. 26, p. 58, 2008.

[5] P. R. D'Argenio, J.-P. Katoen, T. C. Ruys, and J. Tretmans, The bounded retransmission protocol must be on time! Springer, 1997.

[6] H. Lonn and P. Pettersson, "Formal verification of a tdma protocol startup mechanism," in Fault-Tolerant Systems, 1997. Proceedings., Pacific Rim International Symposium on. IEEE, 1997, pp. 235-242.

[7] P. Pettersson, Modelling and verification of real-time systems using timed automata: theory and practice. Citeseer, 1999. 


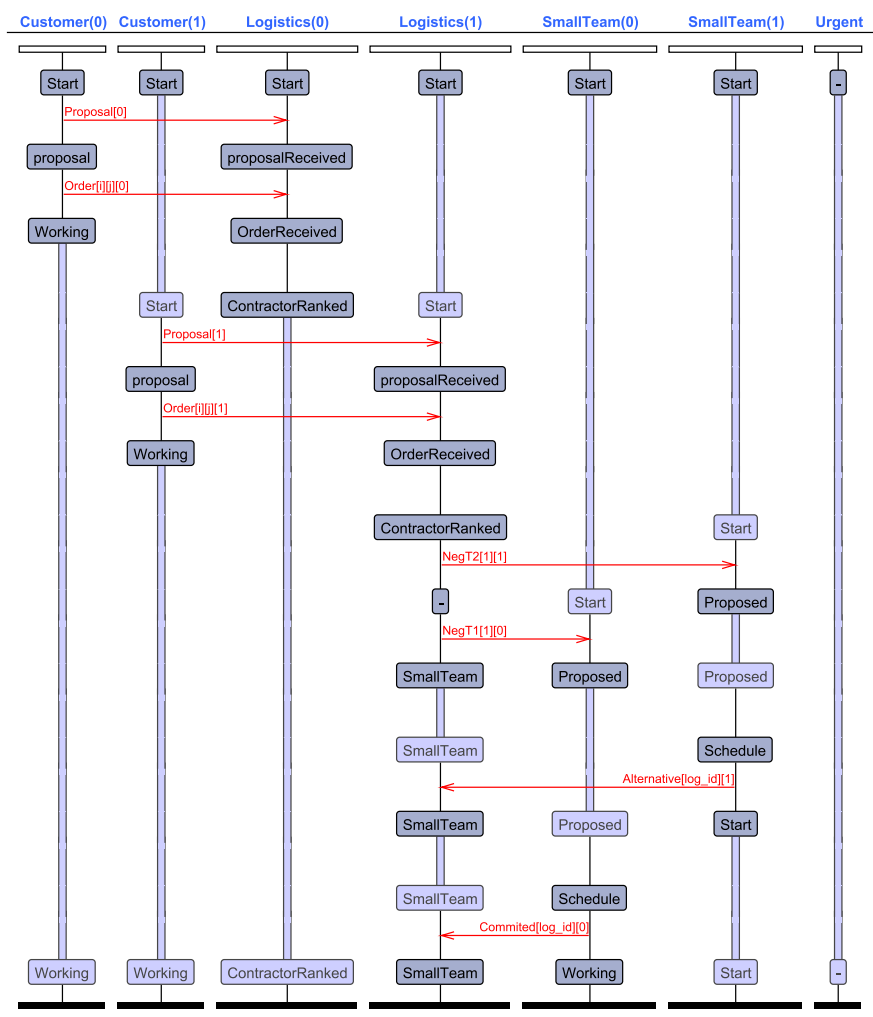

[8] W. Yi, P. Pettersson, and M. Daniels, "Automatic verification of realtime communicating systems by constraint- solving." in FORTE, vol. 6. Citeseer, 1994, pp. 243-258.

[9] M. Atif, "Formal modeling and verification of distributed failure detectors," Faculty of Mathematics and Computer Science, TU/e, vol. 10, 2011.

Fig. 9. Trace for requirement R7. 